\title{
Link-Level Analysis of Downlink Handover Regions in UMTS
}

\author{
Atta Ul Quddus, Xinjie Yang and Rahim Tafazolli, Member, IEEE
}

\begin{abstract}
This paper investigates the downlink handover (soft/softer/hard) performance of Wideband Code Division Multiple Access (WCDMA) based $3^{\text {rd }}$ generation Universal Mobile Telecommunication System (UMTS), as it is known that the downlink capacity of UMTS is very sensitive to the extent of overlap area between adjacent cells and power margin between them. Factors influencing the handover performance such as the correlation between the multipath radio channels of the two links, limiting number of Rake fingers in a handset, imperfect channel estimation, etc. that cannot be modeled adequately in system-level simulations are investigated via link-level simulations. It is also shown that the geometry factor has an influence on the handover performance and exhibits a threshold value (which depends on the correlation between the multipath channels associated with the two links in a handover) above which the performance starts degrading. The variation of the handover gain with the closed loop power control (CLPC) stepsize and space-time transmit diversity (STTD) is also quantified. These comprehensive results can be used as guidelines for more accurate coverage and capacity planning of UMTS networks.
\end{abstract}

Index Terms-UMTS, Code division multi-access, Handover

\section{INTRODUCTION}

$\mathrm{U}$ NIVERSAL Mobile Telecommunication System (UMTS) is currently being deployed and optimised as new services and features are being incorporated. It is necessary to be equipped with accurate air-interface (link-level) and systemlevel simulators to help in the optimisation process. The linklevel simulator provides inputs to the system-level simulator, based on which various performance metrics such as throughput, delay, etc. can be optimised. Some of the techniques that are usually modelled at the system-level such as soft handover ( $\mathrm{SHO}$ ) can also be modelled at the link-level. $\mathrm{SHO}$ is one of the most important functionalities of radio resource management in CDMA based mobile cellular systems. It is used in the intra-frequency handover and allows mobile stations to be connected simultaneously to several base stations, which are selected by a pre-defined SHO algorithm. SHO provides spatial diversity in both the uplink and the downlink, leading to improvements in quality of service. Proper design and tuning of $\mathrm{SHO}$ is one of the main challenges in UMTS, as it has great impact on the trade-offs between coverage, system capacity and service quality in the network.

The impact of SHO is usually investigated through system- level simulations. However, at the system-level, multipath fading is not usually considered, as the pilot channel samples taken for handover purpose are measured on a fairly slow basis and are averaged so as to reduce the number of handovers. Moreover, the use of maximum ratio combining (MRC) in the downlink for SHO users implies that the Rake receiver has an unlimited number of fingers and hence all the multipaths from the base stations involved, can fully contribute to the signal diversity. This is inaccurate, as a practical Rake handset receiver has limited number of fingers [1].

Link-level research on diversity performance, to some extent could rectify these inaccuracies. Realistic multipath channels corresponding to more than one Node-B that are in a handover along with power margin, correctly defined other users' interference and detailed transceiver models can overcome the inaccuracies of SHO performance obtained at system-level. Yet there cannot be found in the literature a comprehensive set of link-level results regarding SHO, softer handover (SerHO) and hard handover (HHO) performance, particularly as a function of the multipath fading correlation between the links. This paper attempts to rectify the omission. Within published literature, the authors in [2] compare the link-level handover gain as a function of the relative path loss between the two Node-Bs, but they model the interference as Gaussian noise, which is a reasonable assumption only for the other-cell interference (caused by distant base stations) and not for the downlink intra-cell interference as well as the interference caused by the adjacent handover cell. In [3], linklevel handover results are presented for four different services in 3GPP Case 3 radio channel but they consider only uncorrelated links with a fixed power margin.

In this paper, we evaluate the performance of $\mathrm{SHO}$ and compare it with that of $\mathrm{HHO}$ and no handover (that models a single cell) for a range of geometry factors (defined as ratio of power received from the serving cell to that from surrounding cells plus the thermal noise) and establish the range of important design parameters that influence the system capacity. The paper is organized as follows: the system model is introduced in section II and the handover performance in section III for a range of geometry factors for a macro as well as a micro cell. Section IV illustrates the impact of various other factors that influence the downlink handover performance. Finally, conclusions are drawn in section V. 


\section{SYSTEM MODEL}

Fig. 1 shows the modelling of the handover process at the link-level. Two Node-Bs are modelled (having independent dedicated physical data channel DPDCH, common pilot channel $\mathrm{CPICH}$ and orthogonal channel noise simulator OCNS that is a mechanism for modelling intra-cell users [4]) as well as the power margin (difference) between the two radio links, an important parameter that triggers the initiation of handover procedure. A desired correlation is introduced between the multipath radio channels from the two Node-Bs. In this paper, we distinguish between $\mathrm{SHO}$ and SerHO based on the correlation between the two links (higher correlation for $\mathrm{SerHO}$ and lower for SHO). In the Rake receiver, fingers are allocated for descrambling, despreading and channel compensation of the signal from both the Node-Bs or only one of them, depending upon whether the UE is in SHO or not.

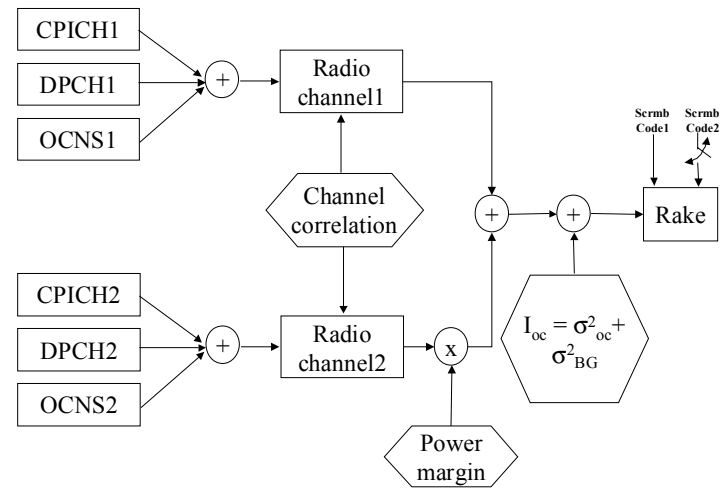

Fig. 1. Concise block diagram of the links from two Node-Bs to one UE

Typically, the multipath fading associated with the two radio links that are in a handover mode is assumed to be uncorrelated which is not always true particularly for SerHO due to the co-location of two sectors of the Node-B. Due to the geometry of the environment, there is a degree of correlation even for the multipath fading between the two radio links. Thus it is important to model the multipath fading correlation as accurately as possible in order to have a true picture of the gains provided by handover. Herein, a method for generating a desired number $N$ of Rayleigh fading envelopes with any desired covariance matrix, as presented in [5], is followed.

The simulation parameters are shown in Table 1 whose values are typical assumptions used for 3GPP simulations $[3,4]$. It should be noted that the 3 GPP Case 1 is a $3 \mathrm{~km} / \mathrm{h}$ twotap channel, representative of a typical urban micro-cell whereas the $3 \mathrm{GPP}$ Case 3 is a $120 \mathrm{~km} / \mathrm{h}$ four-tap channel and represents the propagation characteristics of a fast moving vehicle in a macro-cell [4]. An example of simulator verification is shown in Fig. 2 where our results (marked as UniS) are compared to the results obtained by Nokia [3] for $\mathrm{HHO}$ and SHO and it can be seen that there is a very good match giving credibility to the results presented in the next section.
TABLE 1: SIMULATION PARAMETERS

\begin{tabular}{ll}
\hline \multicolumn{1}{c}{ PARAMETER } & \multicolumn{1}{c}{ ASSUMPTION } \\
\hline Chip Rate & 3.84 Mcps \\
3GPP Reference Channel [4] & $12.2 \mathrm{kbps}$ Speech \\
Radio Channels Used & 3GPP Case 1, 3GPP Case 3 \\
Correlation b/w the 2 Node-B's & Variable between 0.00 to 0.95 \\
Power Diff. b/w the 2 Node-B's & 0 dB or 3 dB \\
Number of Rake Fingers & Variable between 4 and 8 \\
Channel Estimation & Ideal or through CPICH \\
Minimum allowed DPCH $E_{c} / I_{o r}$ & $-45 \mathrm{~dB}$ \\
Maximum allowed DPCH $E_{c} / I_{o r}$ & $-3 \mathrm{~dB}$ \\
Inner Loop Power Control Step Size & Variable b/w 0.5 dB and 2 dB \\
Outer Loop Power Control Step Size & $0.5 \mathrm{~dB}$ \\
Geometry Factor $\left(\hat{I}_{o r} / I_{o c}\right)$ & Variable b/w -6 dB and $+6 \mathrm{~dB}$ \\
Downlink Physical Channels $\&$ & CPICH_Ec/Ior $=-10 \mathrm{~dB}$ \\
Power Levels & DPDCH_Ec/Ior = variable \\
& OCNS_Ec/Ior = power needed \\
\hline \hline
\end{tabular}

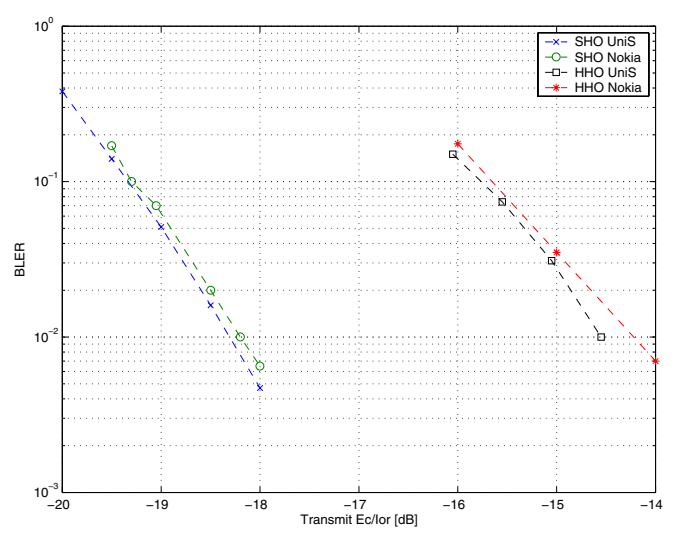

Fig. 2. Comparison with Nokia results [3] in 3GPP Case 3 Channel

\section{IMPACT OF GEOMETRY FACTOR ON HANDOVER}

In Fig. 3, the performance of $\mathrm{NHO}, \mathrm{HHO}$ and $\mathrm{SHO}$ is shown as a function of the geometry factor in 3GPP Case 1 radio channel and in Fig. 4 in the 3GPP Case 3 radio channel.

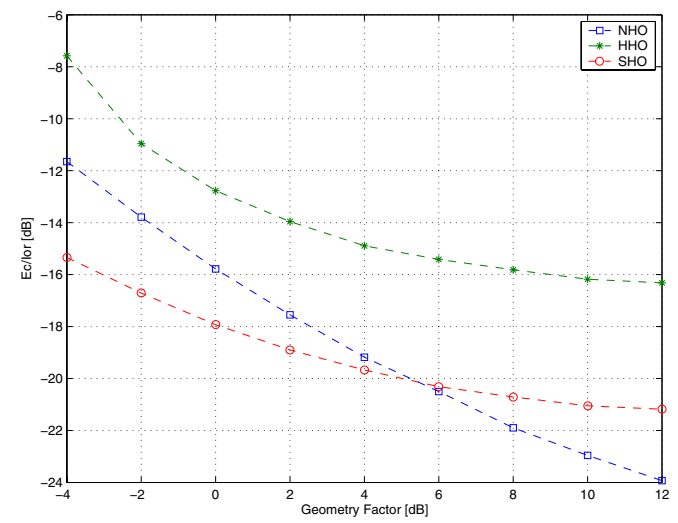

Fig. 3. Performance in Case 1 Radio Channel with Correlation 0

For both sets of results, the power margin between the two 
Node-Bs was $0 \mathrm{~dB}$, i.e. $\left(\hat{I}_{o r 1} / I_{o c}=\hat{I}_{o r 2} / I_{o c}\right)$ and the correlation between the two links was 0 as well. For the Case 1 radio channel, CLPC was switched-on whereas for the Case 3 radio channel, it was switched-off as the Case 3 radio channel corresponds to a mobile velocity of $120 \mathrm{~km} / \mathrm{h}$ and CLPC does not work well at high speeds.

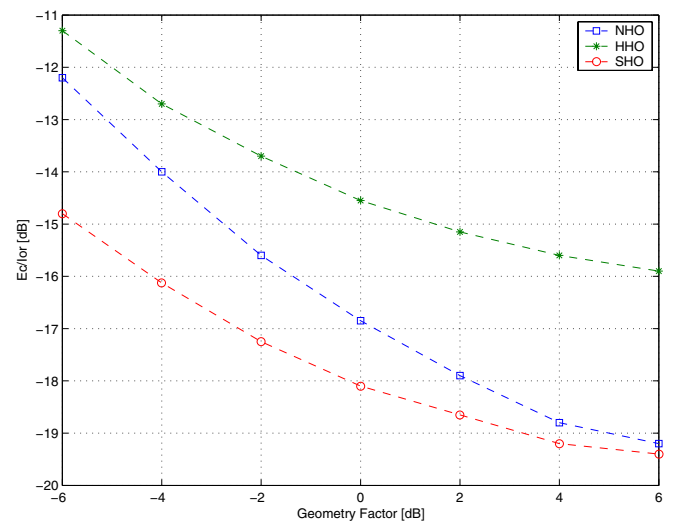

Fig. 4. Performance in Case 3 Radio Channel with Correlation 0

It is interesting to note that in Fig. 3, the performance of SHO becomes worse when compared to NHO for geometry factors greater than or equal to $6 \mathrm{~dB}$. A similar trend is seen in Fig. 4 where at a $6 \mathrm{~dB}$ geometry factor, $\mathrm{NHO}$ and $\mathrm{SHO}$ perform almost identical. The reason for this behaviour is that SHO experiences an interference floor as both the Node-Bs transmit at full power and the scrambling codes are not perfectly orthogonal whereas $\mathrm{NHO}$, which is essentially a single isolated cell, suffers only from the other cells interference $\left(I_{o c}\right)$ and multipath induced interference but not from the interference experienced with the handover. As the geometry factor increases (the other cell interference decreases as in $\hat{I}_{o r} / I_{o c}$ the $\hat{I}_{o r}$ is fixed to unity), the rate of decrease in $E_{c} / I_{o r}$ of SHO is much lower compared to that of NHO. This can be observed in Fig. 3 and Fig. 4 and is due to the fact that the SHO suffers from a multi-access interference floor as pointed out earlier and eventually the $E_{c} / I_{o r}$ of SHO becomes worse than that of NHO. The $6 \mathrm{~dB}$ threshold value at which this happens and causes SHO to suffer a loss in capacity will help in the cell planning as the geometry factor provides a measure of cell isolation.

Since, the multipath fading correlation reduces the signal combining gain, it is expected that with increasing correlation, the SHO $E_{c} / I_{o r}$ will become worse than that of NHO at lower values of geometry factor (i.e. higher levels of the othercells interference). This is illustrated in Fig. 5 for the Case 1 radio channel and in Fig. 6 for the Case 3 radio channel at $1 \%$ BLER and for a very high correlation value of 0.95 . In reality, the multipath fading correlation may not be as high as 0.95 , nevertheless it represents the worst-case scenario. Fig. 5 and Fig. 6 show that the value of the threshold indeed reduces to 2
$\mathrm{dB}$ in the Case 1 radio channel and to $0 \mathrm{~dB}$ in the Case 3 radio channel, as expected. The threshold value of $0 \mathrm{~dB}$ of the geometry factor can be used as a guideline for highly correlated environments.

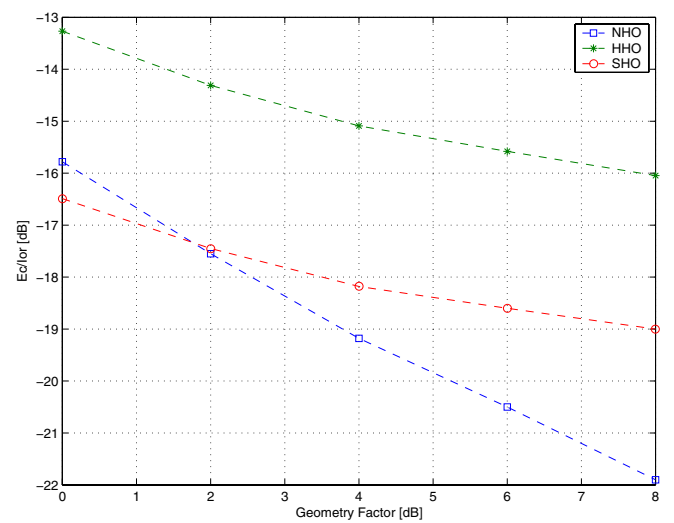

Fig. 5. Performance in Case 1 Radio Channel with Correlation 0.95

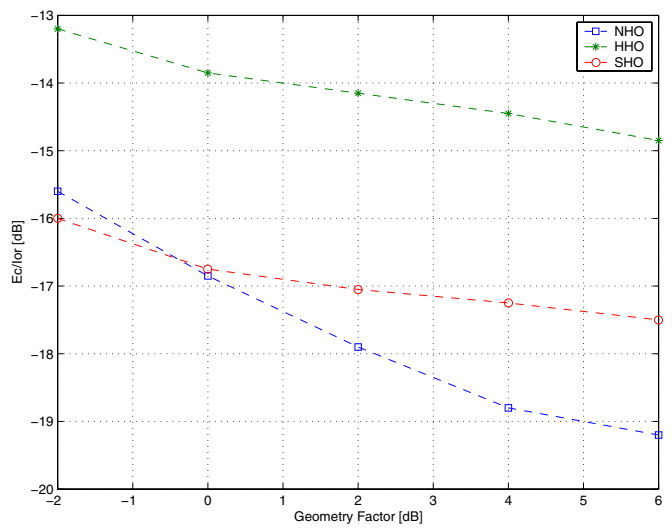

Fig. 6. Performance in Case 3 Radio Channel with Correlation 0.95

\section{Factors Influencing Handover Performance}

In this section, the influence on the handover performance by factors such as the power margin between the two Node-Bs (or two sectors of a Node-B), multipath fading correlation, inner loop power control step size (ILPC), limited number of Rake fingers, channel estimation and STTD are investigated. Note that the SHO Gain in the following results is defined as the difference of $E_{c} / I_{o r}$ values of HHO and SHO at a BLER of $1 \%$. All of the following results were obtained for a geometry factor of $0 \mathrm{~dB}$, a BLER of $1 \%$ and using ideal channel estimation unless otherwise specified.

\section{A. Impact of Correlation and Power Margin}

Table 2 summarizes the $E_{c} / I_{\text {or }}$ values for the SHO and $\mathrm{HHO}$ at $1 \%$ BLER as a function of the multipath fading correlation between the two radio links in 3GPP Case 1 radio channel with the power difference between the two Node-Bs equal to $0 \mathrm{~dB}$ and $3 \mathrm{~dB}$, respectively. For these results, the CLPC was also switched-on. Since the handover is initiated only when the power margin (or power difference) between 
the two Node-Bs is less than or equal to $3 \mathrm{~dB}$, the results of Table 2 represent both the best-case results $(0 \mathrm{~dB}$ power margin) and worst-case results (3 dB power margin).

TABLE 2: CORRELATION AND POWER MARGIN IMPACT IN CASE 1 CHANNEL

\begin{tabular}{|c|c|c|c|c|c|c|}
\hline \multirow[b]{2}{*}{ 兽 } & \multicolumn{3}{|c|}{ Power Margin $=0 \mathrm{~dB}$} & \multicolumn{3}{|c|}{ Power Margin $=3 \mathrm{~dB}$} \\
\hline & $\begin{array}{c}\mathrm{SHO} \\
E_{c} / I_{o r} \\
{[\mathrm{~dB}]}\end{array}$ & $\begin{array}{c}\mathrm{HHO} \\
E_{c} / I_{o r} \\
{[\mathrm{~dB}]}\end{array}$ & $\begin{array}{l}\text { Gain } \\
{[\mathrm{dB}]}\end{array}$ & $\begin{array}{c}\mathrm{SHO} \\
E_{c} / I_{o r} \\
{[\mathrm{~dB}]}\end{array}$ & $\begin{array}{c}\mathrm{HHO} \\
E_{c} / I_{o r} \\
{[\mathrm{~dB}]}\end{array}$ & Gain $[\mathrm{dB}]$ \\
\hline 0.00 & -17.93 & -12.77 & 5.16 & -16.95 & -14.30 & 2.65 \\
\hline 0.20 & -17.70 & -13.08 & 4.62 & -16.71 & -14.21 & 2.50 \\
\hline 0.40 & -17.38 & -13.22 & 4.16 & -16.52 & -14.25 & 2.27 \\
\hline 0.60 & -17.12 & -13.32 & 3.80 & -16.32 & -14.26 & 2.06 \\
\hline 0.80 & -16.74 & -13.24 & 3.50 & -15.88 & -14.20 & 1.68 \\
\hline 0.95 & -16.49 & -13.26 & 3.22 & -15.69 & -14.26 & 1.42 \\
\hline
\end{tabular}

It is seen from Table 2 that a significant SHO gain of 5.16 $\mathrm{dB}$ is obtained at a correlation of 0 when the power margin is $0 \mathrm{~dB}$. By increasing the correlation, the gain steadily reduces and at a correlation of 0.95 , the gain has reduced by $1.94 \mathrm{~dB}$, i.e. $(5.16-3.22=1.94)$. Moreover since the SerHO usually experiences high values of correlation due to the co-location of the two sectors of a Node-B whereas SHO typically experiences low or almost no correlation due to the physical separation of the two Node-Bs, SerHO is expected to support $28 \%=\left(1-10^{(-17.93-(-16.49)) / 10}\right)$ less users than SHO. Table 2 also shows that when the power margin between the two Node-Bs is $3 \mathrm{~dB}$, SHO performs about $1 \mathrm{~dB}=(-16.95+17.93)$ worse as compared to $0 \mathrm{~dB}$ power margin, but the $\mathrm{HHO}$ performs $1.53 \mathrm{~dB}=(-14.3+12.77)$ better than the $\mathrm{HHO}$ performance with $0 \mathrm{~dB}$ power margin. This is logical since in $\mathrm{HHO}$, the signal from the second Node-B is not combined and acts as an interferer, and the lower the interference power, the better the performance. Because of this, the SHO gain over the $\mathrm{HHO}$ also reduces significantly with the increasing power margin, e.g. at correlation of 0 the $\mathrm{SHO}$ gain reduces from $5.16 \mathrm{~dB}$ to $2.65 \mathrm{~dB}$. Table 3 shows the impact of the correlation and the power margin on the $\mathrm{HHO}$ and the $\mathrm{SHO}$ performance in the $3 \mathrm{GPP}$ Case 3 radio channel with CLPC disabled.

TABLE 3: CORRELATION AND POWER MARgIN IMPACT IN CASE 3 CHANNEL

\begin{tabular}{|c|c|c|c|c|c|c|}
\hline \multirow[b]{2}{*}{ 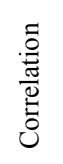 } & \multicolumn{3}{|c|}{ Power Margin $=0 \mathrm{~dB}$} & \multicolumn{3}{|c|}{ Power Margin $=3 \mathrm{~dB}$} \\
\hline & $\begin{array}{l}\mathrm{SHO} \\
E_{c} / I_{o r} \\
{[\mathrm{~dB}]}\end{array}$ & $\begin{array}{l}\mathrm{HHO} \\
E_{c} / I_{o r} \\
{[\mathrm{~dB}]}\end{array}$ & $\begin{array}{l}\text { Gain } \\
{[\mathrm{dB}]}\end{array}$ & $\begin{array}{l}\mathrm{SHO} \\
E_{c} / I_{o r} \\
{[\mathrm{~dB}]}\end{array}$ & $\begin{array}{l}\mathrm{HHO} \\
E_{c} / I_{o r} \\
{[\mathrm{~dB}]}\end{array}$ & $\begin{array}{l}\text { Gain } \\
{[\mathrm{dB}]}\end{array}$ \\
\hline 0.00 & -18.20 & -14.55 & 3.65 & -17.50 & -15.60 & 1.90 \\
\hline 0.20 & -17.80 & -14.30 & 3.50 & -17.10 & -15.45 & 1.65 \\
\hline 0.40 & -17.45 & -14.05 & 3.40 & -16.85 & -15.35 & 1.50 \\
\hline 0.60 & -17.00 & -13.85 & 3.15 & -16.40 & -15.20 & 1.20 \\
\hline 0.80 & -16.75 & -13.85 & 2.90 & -16.20 & -15.20 & 1.00 \\
\hline 0.95 & -16.75 & -13.85 & 2.90 & -16.10 & -15.20 & 0.90 \\
\hline
\end{tabular}

A closer observation of Table 2 and Table 3 reveals that the performance of both $\mathrm{SHO}$ and HHO is improved in the $3 \mathrm{GPP}$ Case 3 channel compared to the 3GPP Case1 channel due to availability of more multipath diversity and a greater degree of time variations in the former. However, the improvements to $\mathrm{SHO}$ are less as compared to $\mathrm{HHO}$, as $\mathrm{SHO}$ combining has already taken advantages of channel diversity, leading to lower SHO gains in the 3GPP Case3 channel. Also in Case 3, the relative performance loss of SerHO as compared to the SHO is similar to that in Case 1 radio channel, i.e. $28 \%$ $=\left(1-10^{(-18.20-(-16.75)) / 10}\right)$, at a power margin of $0 \mathrm{~dB}$. Thus the multipath fading correlation affects the relative SHO performance similarly in the two fading channels.

\section{B. Impact of ILPC Step Size}

Table 4 shows the impact of the inner loop power control (ILPC) step size on the handover performance for a power margin of $0 \mathrm{~dB}$ and $3 \mathrm{~dB}$, respectively. The correlation is assumed to be 0 for these results.

TABLE 4: IMPACT OF ILPC STEP SIZE IN 3GPP CASE 1 RADIO CHANNEL

\begin{tabular}{ccccccc}
\hline \hline & \multicolumn{3}{c}{ Power Margin $=0 \mathrm{~dB}$} & \multicolumn{3}{c}{ Power Margin $=3 \mathrm{~dB}$} \\
\cline { 2 - 7 } $\begin{array}{c}\text { Step } \\
\text { Size } \\
{[\mathrm{dB}]}\end{array}$ & $\begin{array}{c}\mathrm{SHO} \\
E_{c} / I_{\text {or }} \\
{[\mathrm{dB}]}\end{array}$ & $\begin{array}{c}E_{c} / I_{\text {or }} \\
{[\mathrm{dB}]}\end{array}$ & $\begin{array}{c}\text { Gain } \\
{[\mathrm{dB}]}\end{array}$ & $\begin{array}{c}E_{c} / I_{\text {or }} \\
{[\mathrm{dB}]}\end{array}$ & $\begin{array}{c}E_{c} / I_{\text {or }} \\
{[\mathrm{dB}]}\end{array}$ & $\begin{array}{c}\text { Gain } \\
{[\mathrm{dB}]}\end{array}$ \\
\hline 0.50 & -17.91 & -11.91 & 6.00 & -16.91 & -13.44 & 3.47 \\
1.00 & -17.93 & -12.77 & 5.16 & -16.95 & -14.30 & 2.65 \\
2.00 & -17.90 & -13.05 & 4.85 & -16.99 & -14.41 & 2.58 \\
\hline \hline
\end{tabular}

It is seen that $\mathrm{SHO} E_{c} / I_{\text {or }}$ does not vary much as the stepsize changes from $0.5 \mathrm{~dB}$ to $2 \mathrm{~dB}$, whereas $\mathrm{HHO} E_{c} / I_{\text {or }}$ improves. This is due to the fact that for $\mathrm{HHO}$ a larger power control step-size also helps overcome the interference caused by the signal from the second Node-B that acts as an interferer and is not used for signal combining. On the other hand, SHO, due to signal combining from the two Node-Bs is seen to be relatively independent of ILPC step-size in Case 1 channel.

TABLE 5: IMPACT OF ILPC STEP SIZE IN 3GPP CASE 3 RADIO CHANNEL

\begin{tabular}{ccccccc}
\hline \hline & \multicolumn{3}{c}{ Power Margin $=0 \mathrm{~dB}$} & \multicolumn{3}{c}{ Power Margin $=3 \mathrm{~dB}$} \\
\cline { 2 - 7 } $\begin{array}{c}\text { Step } \\
{[\mathrm{dB}]}\end{array}$ & $\begin{array}{c}\text { SHO } \\
E_{c} / I_{\text {or }} \\
{[\mathrm{dB}]}\end{array}$ & $\begin{array}{c}E_{c} / I_{\text {or }} \\
{[\mathrm{dB}]}\end{array}$ & $\begin{array}{c}\text { Gain } \\
{[\mathrm{dB}]}\end{array}$ & $\begin{array}{c}E_{c} / I_{\text {or }} \\
{[\mathrm{dB}]}\end{array}$ & $\begin{array}{c}\text { HHO } \\
E_{c} / I_{o r} \\
{[\mathrm{~dB}]}\end{array}$ & $\begin{array}{c}\text { Gain } \\
{[\mathrm{dB}]}\end{array}$ \\
\hline 0.50 & -17.11 & -13.87 & 3.24 & -16.57 & -14.74 & 1.83 \\
1.00 & -17.18 & -13.79 & 3.39 & -16.70 & -14.85 & 1.85 \\
2.00 & -16.99 & -13.45 & 3.54 & -16.34 & -14.39 & 1.95 \\
\hline \hline
\end{tabular}

Table 5 shows the performance in the 3GPP Case 3 radio channel and it can be seen that the SHO $E_{c} / I_{\text {or }}$ shows an optimum step-size of $1 \mathrm{~dB}$ for a power margin of $0 \mathrm{~dB}$ as well as $3 \mathrm{~dB}$. The performance degrades at higher step sizes (e.g. 2 $\mathrm{dB})$ due to the faster variations of the $120-\mathrm{km} / \mathrm{h}$ radio channel. Again, the HHO is seen to perform relatively better in the Case 3 than in the Case 1 radio channel. It is also noted from 
Table 5 and Table 3 that in the Case 3 radio channel, by enabling the CLPC, the SHO performs worse $\left(E_{c} / I_{o r}=-\right.$ $17.18 \mathrm{~dB}$ ) at a step-size of $1 \mathrm{~dB}$ as compared to the $\mathrm{SHO}$ with CLPC disabled $\left(E_{c} / I_{o r}=-18.20 \mathrm{~dB}\right)$. Thus at high speeds, the CLPC should not be used, at least from the perspective of SHO.

\section{Impact of Channel Estimation}

Since, channel estimation (CHEST) is more challenging at high speeds, we present the impact of CHEST through the $\mathrm{CPICH}$ only in the 3 GPP Case 3 radio channel having 120 $\mathrm{km} / \mathrm{h}$ mobile velocity. Table 6 illustrates the impact of CHEST on the $E_{c} / I_{\text {or }}$ of $\mathrm{NHO}, \mathrm{HHO}$ and $\mathrm{SHO}$ at $1 \%$ BLER, 0 correlation and the power margin equal to $0 \mathrm{~dB}$. It is observed that estimating the channel through $\mathrm{CPICH}$ incurs a loss in $E_{c} / I_{o r}$ of about $0.3-0.5 \mathrm{~dB}$. Note that the channel estimation method that has been used in the simulations is a simple pilot averaging method. With more sophisticated methods, the $11 \%=\left(1-10^{-0.5 / 10}\right)$ loss in capacity of SHO can be reduced still further.

TABLE 6: IMPACT OF CHEST IN 3GPP CASE 3 CHANNEL WITH CLPC OFF

\begin{tabular}{cccc}
\hline \hline$E_{c} / I_{\text {or }}[\mathrm{dB}]$ & Ideal CHEST & $\begin{array}{c}\text { Through } \\
\text { CPICH }\end{array}$ & Loss $[\mathrm{dB}]$ \\
\hline NHO & -16.85 & -16.50 & 0.35 \\
HHO & -14.55 & -14.10 & 0.45 \\
SHO & -18.20 & -17.70 & 0.50 \\
\hline \hline
\end{tabular}

\section{Impact of the Number of Rake Fingers}

A typical Rake receiver in a UE has four to six Rake fingers. Since, for SHO in the 3GPP Case3 channel, the total number of multipaths is 8 , the impact of limited number of Rake fingers is shown in Table 7 by assuming a correlation of 0 between the two links and CLPC switched off.

TABLE 7: IMPACT OF NO. OF RAKE FINGERS IN CASE 3 CHANNEL

\begin{tabular}{crc}
\hline \multirow{2}{*}{ No. of Fingers } & Power Margin $=0 \mathrm{~dB}$ & Power Margin $=3 \mathrm{~dB}$ \\
\cline { 2 - 3 } & SHO $E_{c} / I_{o r}[\mathrm{~dB}]$ & SHO $E_{c} / I_{o r}[\mathrm{~dB}]$ \\
\hline 8 & -18.20 & -17.50 \\
6 & -18.10 & -17.40 \\
4 & -17.90 & -17.30 \\
\hline \hline
\end{tabular}

Note that for these results, the fingers are allocated to the paths having the highest power in descending order. Due to this finger allocation mechanism, with 4 Rake fingers, the $E_{c} / I_{\text {or }}$ of SHO suffers at most by $0.3 \mathrm{~dB}=(-17.90+18.20)$, i.e. a loss of $7 \%=\left(1-10^{-0.3 / 10}\right)$ in capacity, in the 3GPP Case 3 channel, when the power margin is $0 \mathrm{~dB}$.

\section{E. Impact of STTD}

The impact of STTD on the SHO performance is illustrated in Table 8 for 3GPP Case 1 radio channel with CLPC enabled and a correlation value of 0 . Note that in Table 8 , the STTD gain is the gain provided by STTD in SHO and not the gain provided by SHO over the HHO performance. It is seen that the relative gain provided by STTD in SHO is around $0.5 \mathrm{~dB}$. In [6], it is reported that STTD provides a gain of $0.8 \mathrm{~dB}$ in a typical micro-cell without taking into account handover, thus the $0.3 \mathrm{~dB}$ reduction $(0.8-0.5)$ in the STTD gain in SHO seems logical as the SHO already provides a degree of diversity and the additional diversity gain provided by STTD is expected to be somewhat less.

TABLE 8: IMPACT OF STTD IN 3GPP CASE 1 CHANNEL WITH CLPC ON

\begin{tabular}{|c|c|c|c|}
\hline \multirow{2}{*}{$\begin{array}{c}\text { Power } \\
\text { Margin } \\
{[\mathrm{dB}]}\end{array}$} & \multicolumn{2}{|c|}{$\mathrm{SHO} E_{c} / I_{o r}[\mathrm{~dB}]$} & \multirow{2}{*}{$\begin{array}{l}\text { STTD Gain } \\
{[\mathrm{dB}]}\end{array}$} \\
\hline & With STTD & Without STTD & \\
\hline 0 & -18.46 & -17.93 & 0.53 \\
\hline 3 & -17.42 & -16.95 & 0.47 \\
\hline
\end{tabular}

\section{CONCLUSION}

In this paper, the performance of (soft/softer/hard) handover in WCDMA based UMTS FDD downlink is analysed at the link-level in 3GPP Case 1 and 3GPP Case 3 radio channels. It is shown that there is a trade-off between the signal combining gain provided by the handover, the multi-access interference associated with the handover and the other cells interference. Threshold values of the geometry factor are established beyond which the performance of SHO becomes worse than that of a single isolated cell. Factors influencing the handover performance such as the multipath fading correlation, power control step-size, channel estimation as well as transmit antenna diversity, are investigated and their impact on the handover is illustrated in terms of the average transmit power. These link-level results will help in the system-level analysis and provide guidelines for UMTS network dimensioning.

\section{REFERENCES}

[1] A. Chheda, "A Performance Comparison of the CDMA IS-95B and IS95A Soft Handoff Algorithms", in Proc. IEEE VTC, Houston, TX, vol. 2 , pp. $1407-1412,16^{\text {th }}-20^{\text {th }}$ May 1999.

[2] O. Salonaho and J. Laakso, "Flexible Power Allocation For Physical Control Channel in Wideband CDMA", in Proc. IEEE VTC, Houston, TX, pp. 1455 - 1458, May 1999.

[3] Nokia, "Inter cell soft handover simulation results for UE performance tests", 3GPP TSG-RAN WG4 Meeting \#9, TSGR4\#9(99)805, Bath, UK, December $7^{\text {th }}-10^{\text {th }}, 1999$.

[4] 3GPP TSG RAN, "User Equipment (UE) radio transmission and reception (FDD)", Release 6, TS-25.101, V8.0.0 (2007-09).

[5] B. Natarajan, C. R. Nassar, and V. Chandrasekhar, "Generation of Correlated Rayleigh Fading Envelopes for Spread Spectrum Applications", IEEE Commun. Lett., vol. 4, no. 1, pp. 9-11, Jan. 2000

[6] H. Holma and A. Toskala, WCDMA For UMTS - Radio Access For Third Generation Communications, $3^{\text {rd }}$ Ed., Chichester: John Wiley \& Sons Ltd, 2004. 